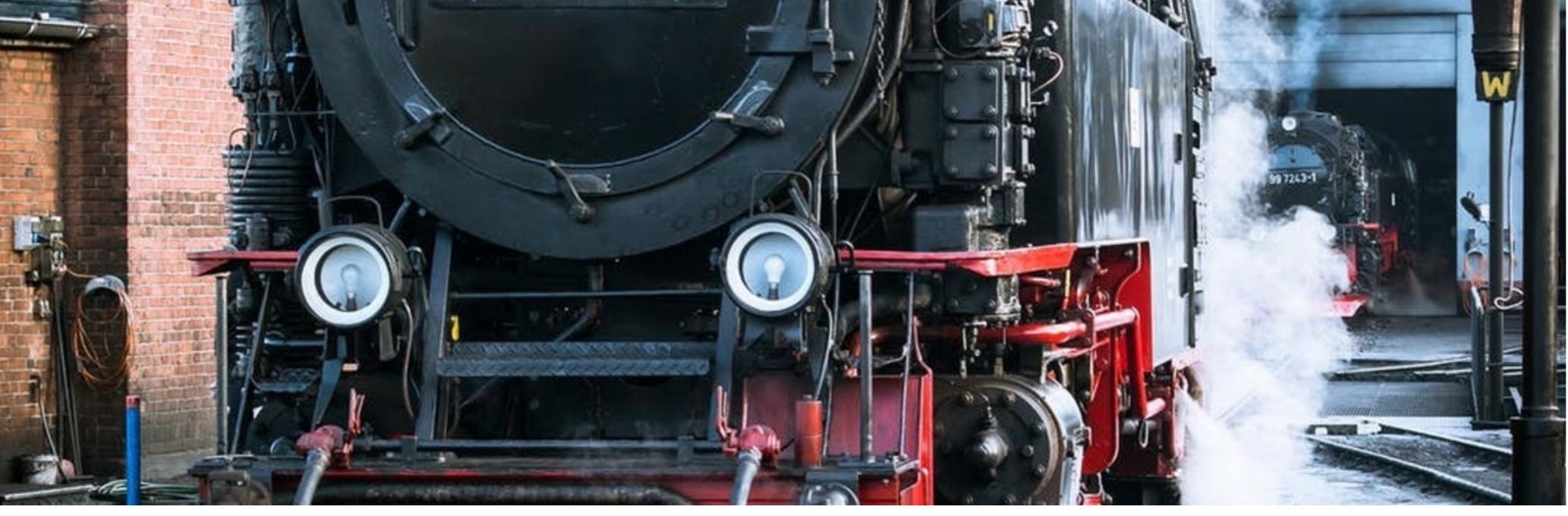

\title{
ТЕХНИЧЕСКИЕ СРАВНЕНИЯ МЕЖДУ УЗКОЙ ШИРИНОЙ КОЛЕИ И СТАНДАРТОМ - Technical Comparisons between narrow Gauge and standard for electric Trains in Bogota
}

Authors: Juan Pablo Escobar Fernandez, Juan Pablo Escobar Fernandez

Submitted: 15. June 2020

Published:

22. June 2020

Volume: 7

Issue: 3

Affiliation: Languages: Keywords:

Categories: RUDN University, Moscow, Russia Russian Railway, electric Train, Gauge, Bogota, technical comparisons, track width.

DOl: $\quad$ 10.17160/josha.7.3.683

Abstract:

Life Sciences
10.17160/josha.7.3.683

For decades, rail track width measures have been discussed that allow for higher rail speed, greater stability, and safety of railcars. The city of Bogota is no exception, and this is the topic being discussed for the construction of an electric train that would link the city of Bogota with its surroundings. Tasks: For that reason, in this article we will develop a comparison between the two most common types of measurements used on a railway for an electric train. We will analyze the technical data in theory and get help from the experience of railway work from other cities. Hypothesis: We will also analyze two hypotheses that arise to choose the type of track that best suits the city of Bogota. One is to choose a narrow track or \&quot;yard\&quot; that is commonly used in tram and light rail. Another hypothesis is the choice of a standard or international sidewalk, which is used in most intercity trains and metro. Method and Result:

\section{JOSHA \\ Journal of Science, \\ Humanities and Arts




\section{ТЕХНИЧЕСКИЕ СРАВНЕНИЯ МЕЖДУ УЗКОЙ ШИРИНОЙ КОЛЕИ И СТАНДАРТОМ ДЛЯ ЭЛЕКТРИЧЕСКАЯ ПОЕЗДА В ГОРОДЕ БОГОТА}

\section{TECHNICAL COMPARISONS BETWEEN NARROW GAUGE AND STANDARD FOR ELECTRIC TRAINS IN BOGOTA}

\section{By Juan Pablo Escobar, masters student, Peoples' Friendship University of Russia, Moscow}

Abstract. Objective: For decades, rail track width measures have been discussed that allow for higher rail speed, greater stability and safety of railcars. The city of Bogota is no exception, and this is the topic being discussed for the construction of an electric train that would link the city of Bogota with its surroundings. Tasks: For that reason, in this article we will develop a comparison between the two most common types of measurements used on a railway for an electric train. We will analyze the technical data in theory and get help from the experience of railway work from other cities. Hypothesis: We will also analyze two hypotheses that arise to choose the type of track that best suits the city of Bogota. One is to choose a narrow track or "yard" that is commonly used in tram and light rail. Another hypothesis is the choice of a standard or international sidewalk, which is used in most intercity trains and metro. Method and Result: Qualitative social survey method was adopted. We will demonstrate with historical data, formulas, calculations and engineering criteria which of the two track widths is best suited for the city Bogota.

Keywords: Railway, electric train, gauge, Bogota, technical comparisons, track width. 


\section{JOSHA}

Journal of Science, Humanities and Arts

Аннотация. Цель: На протяжении десятилетий обсуждались меры ширины железнодорожных путей, которые позволяют развивать более высокую железнодорожную скорость, большую стабильность и безопасность вагонов. Город Богота не является исключением, и именно эта тема обсуждается для строительства электропоезда, который связывал бы город Богота с его окрестностями. Задачи: Вот поэтому в этой статье мы разработаем сравнение между двумя наиболее распространенными типами измерений, используемых на железной дороге для электропоезда. Мы проанализируем технические данные в теории и получим помощь от опыта железнодорожных работ из других городов. Гипотеза: Мы также обсудим две гипотезы, которые возникают, чтобы выбрать тип колеи, который лучше всего подходит для города Богота. Один из них: выбрать узкую колею или "ярд", которая обычно используется на трамваях и легких железных дорогах. Другой гипотезой является выбор стандартного или международного тротуара, который используется в большинстве поездов и метро. Методы и результаты: Использован метод качественного социального опроса. Мы продемонстрируем с историческими данными, формулами, расчетами и инженерным критерием, какая из двух ширины колеи лучше всего подходит для города Богота.

Ключевые слова: Железная дорога, электропоезд, колей, Богота, технические сравнения, ширина колеи. 


\section{Введение}

Когда первые стальные рельсы были введены в железнодорожные пути в середине XIX века, железнодорожному транспорту было признано большое значение, которое он имеет как средство связи. "Сочетание двух элементовсамонаводящихся и движущей силы-было вызвано английским инженером Ричардом Тревитиком, когда 24 февраля 1804 года Локомотив проехал 5 вагонов, груженных 10 тоннами стали и 70 людьми, на расстоянии 15 километров, разделяющем деревни Пеннидаран и Аберкинонв Уэльсе .’[1]

“Принцип железной дороги основан на осях, которые катятся по рельсам, удерживаемым жесткими элементами, называемыми шпалами, которые передают грузы на Землю через заполняющую подушку (щебень и просеянная порода), называемую балластом.” [2] Этот фундамент остается неизменным с течением времени, несмотря на большие изменения скоростей, нагрузок на ось, автоматики эксплуатации и качества подвижного состава сборки.

Так же, как они имеют огромные преимущества, железная дорога имеет свои ограничения, как следствие ее двух основных характеристик:

- Натирание металла на металле: колесо на дорожке.

- Поддержание определенных траекторий, ограничение свободы.

Низкое трение металла на металле (3 кг/тонна) позволяет мобилизовать тысячи тонн с уменьшенной мощностью и умеренным потреблением энергии. Эта же функция требует остановочных расстояний, которые увеличиваются со скоростью и требуют экстремальной сигнализации и тормозных систем, а также ограничения склонов на участках наибольшего транзита. 
Поддержание точной траектории позволяет эффективно использовать платформу с экономией пространства и уменьшенными галибами, что приводит, среди прочего, к некоторым преимуществам:

- Способность перевозить людей и товары

- Простота автоматизации и применения современных вычислений

- Безопасность на очень высоких скоростях

- Независимость платформы, которая предотвращает перегрузку

\section{Сравнительное техническое исследование между узкой шириной колеи и}

\section{стандартом}

Анализ технических и эксплуатационных параметров был основан на предположении о предполагаемом спросе на грузы в 2000 году, оцениваемом в 3-5 млн. тонн в год, и гипотетическом увеличении общего грузопотока.

Основные данные независимы от альтиметрической плоской конструкции линии.

Основные параметры:

- Тип рельса и поддерживаемый вес на ось

- Тип и характеристикы тележки

- Тип, характеристика и тяговая способность локомотивов

- Тип рельса и поддерживаемый вес на ось:

В настоящее время и в случае линий, пересекающих Саванну Боготы, они оснащены рельсами 75 фунтов/Ярд и с максимальным допустимым весом на ось 16 тонн. Пригородные поезда в Европе со ссылкой на правила Международного союза железных дорог (U.I.E.) приняли профильные рельсы 100Y 120lbs/Ярд для максимальных весов, поддерживаемых на ось 20 и 25 тонн для конструкций. 
Типи особенность пригородный поезд (включая тяговую тележку ):

Хотя они являются переменными для каждого типа проекта и спроса на обслуживание, в целом основные характеристики можно суммировать следующим образом: длина ветки, состоящей из двух моторных тележек и двух прицепов $=104$ метра, вес ветки $=205$ тонн, мощность.

одна ветка $=2800$ кВт, максимальная скорость $=140$ км/ч, пассажировместимость, начиная с индекса комфорта 4 человека / м2, 843 пассажира.

\section{Основные данные, которые зависят от альтиметрической плоской}

\section{конструкции линии}

Скорость поездов, их состав и протяженность, отношение тары: 1 вес перевозимых пассажиров, а также пропускная способность линии варьируются в зависимости от плоско - альтиметрических характеристик de la пути и типа принятого тротуара.

Наклон дорожек, пересекающих Саванну, в среднем не более 2\%; значения радиусов поворотов превышают в среднем 500 метров, что обеспечивает широкие скорости.

Максимальная расчетная скорость линии, гарантирующая приемлемое качество движения, напрямую зависит от значения радиуса, виража и неподъемного бокового ускорения, поддерживаемого для типов ветвей, которые будут циркулировать. Как видно выше, общепринятая формула выглядит следующим образом:

$\mathrm{V}$ максима $=\mathrm{K}$

Для стандартного колея значение универсально принятого коэффициента К составляет 4,6 при вираже 15-16 см и несмещенном боковом ускорении 0,6 
м/сек2, согласно стандарту U. I. Е. Для узкая колея из- за отсутствия определенных правил значение коэффициента $\mathrm{K}$ составляет 3.36 , исходя из гипотезы о том, что процентное изменение нагрузки между колесом и рельсом было одинаковым для обоих тросов во всех условиях нагрузки.

\section{Сопротивление к подаче в-прямой и изогнутой}

Для расчета сопротивления вперед, в плоскости и в прямом, в среднем на единицу веса, выраженную в кг / тонне, используется формула Дэвиса для всей ветви, то есть транспортных средств прицепов и тракторных транспортных средств, принимая во внимание оба типа тротуара. Уравнения для каждого случая выражаются следующим образом:

Для узкая колея:

$\mathrm{r}=2.77+0.03 \mathrm{~V}+0.00035 \mathrm{~V} 2[3]$

Для стандартного колея:

$\mathrm{r}=2.77+0.014 \mathrm{~V}+0.0003 \mathrm{~V} 2[4]$

В зависимости от типа поездов, движущихся по стандартной или дворовой тропе и для разных радиусов, результат применения приведенного выше уравнения суммируется следующим образом.

Таблица 1. Максимальная скорость на повороте

\begin{tabular}{|c|c|c|c|c|c|c|}
\hline Oписание & \multicolumn{3}{|c|}{ Колея ярд } & \multicolumn{3}{|c|}{ Колея стандартная } \\
\hline \multirow{6}{*}{$\begin{array}{c}\text { максимальная скорость на } \\
\text { повороте } \mathrm{V}=\mathrm{k} * \mathrm{R}\end{array}$} & $\mathbf{K}$ & $\mathbf{R}(\mathbf{m})$ & $\mathrm{V}(\mathrm{km} / \mathrm{h})$ & $\mathbf{K}$ & $\mathbf{R}(\mathbf{m})$ & $\mathrm{V}(\mathrm{km} / \mathrm{h})$ \\
\hline & \multirow{5}{*}{3.36} & 150 & 40 & \multirow{5}{*}{4.6} & 150 & 50 \\
\hline & & 200 & 45 & & 200 & 65 \\
\hline & & 250 & 53 & & 250 & 73 \\
\hline & & 300 & 58 & & 300 & 79 \\
\hline & & 500 & 75 & & 500 & 103 \\
\hline
\end{tabular}

Источник: исследование Carare Railway. 1980 год 
Тяговая способность и скорость режима наклона (подъема)рассчитываются от максимальной мощности тракторных тележек до предела сцепления. Часть других переменных, таких как скорость и смещенный наклон, выраженный в процентах.

Оценка интенсивности движения или поездов /дней по линии опережает гипотезы спроса на пассажирские перевозки (может быть в год) и прогноз будущего трафика на 15 лет. Известно, что расстояние между станциями пересечения и относительное время в пути являются основными параметрами, определяющими пропускную способность линии в поездах/день, существует несколько выражений, таких как" школы", которые позволяют рассчитать ее.

Устанавливая альтиметрические характеристики линии и принимая критические радиусы, которые наиболее подходят для морфологии маршрута, рассчитывается средняя скорость для обоих направлений и всех поездов, движущихся по этому пути. Предполагается, чтов случае пригородного поезда Боготы (один)путь должен быть разделен между грузовыми и пассажирскими поездами, что является достаточной причиной для тщательного выполнения расчетов.

Выбор типа колея, которая будет использоваться для железной дороги, удовлетворяющей спрос на транзит, имеет решающее значение с точки зрения геометрических характеристик и, в частности, выбора расстояний между станциями пересечения и их протяженностью.

\section{Проблема железной дороги в Боготе}


Как видно выше, вход северных и западных железнодорожных коридоров на станцию Саванна осуществляется через городскую ткань Боготы. Эта ситуация привела к одной из самых серьезных проблем в его эксплуатации, из-за пересечения уровня с улицами, гонками и проспектами, мешая движению по городу. Этот конфликт начался с роста Боготы, который не был ориентиром для старых железнодорожных путей; напротив, рельсы привели к дискомфорту и введению на уровне в зависимости от городских районов , которые предполагали связь между автомобилем или автобусом.

Несколько скептически относясь к существенному увеличению нагрузки, но также не зная о том, что объем (в среднесрочной и долгосрочной перспективе) в 1000000 тонн / год, что эквивалентно 1670 поездам / год / направление, будет означать в среднем 34 поездам в неделю / направление. Этот факт делает очень труднымсовместное использование коридоров с массивной системой пассажиров на станцию Саванна и от нее, что потребует, для привлекательной работы спроса, иметь менее 17 часов непрерывного обслуживания.

Таким образом, выдвигаются три альтернативы для решения вопроса о работе двух одновременных грузовых и пассажирских услуг при сохранении единого пути:

- Построить вариант для западного сектора, используя, например, коридор A. L. O, чтобы освободить нынешние коридоры и посвятить их исключительно пригородным поездам.

- Оградить от национальной железной дороги участок, ранее известный как" железная дорога Кундинамарка " между Facatativá и Пуэрто-Сальгар и 
работать по дороге туда и обратно, груз, который приходит или берет свое начало в Боготе.

- Совместное использование одного и того же коридора; однако возникает проблема технологических различий между подвижным составом, который имеет железные дороги для перемещения грузов, и тем, который требует современного обслуживания пассажиров.

\section{Заключение}

Что касается движения поездов в городской структуре, то, если проект был выгоден для концессионера, следует принять во внимание одобрение мастерской общественного пространства по смягчению негативного воздействия, которое могли бы оказать перепады (мосты или box coulverts), закрытие железнодорожных переездов и остановочных пунктов.

Инвестиции, требуемые от столичного округа, должны быть обеспечены высоким показателем мобильности пассажиров, и эта система должна быть интегрирована в программу «Transmilenio». Однако неудача из-за низкого спроса пользователей не исключена.

Путем краткой оценки каждой альтернативы можно найти наиболее приемлемое решение проблемы, если исходить из того, что ширина нынешнего железного коридора недостаточна, а материалы надстройки устарели и что по причинам, изложенным в анализе ширины тротуара, необходимо вставить стандартную колею 1435 мм. 


\section{Список литературы}

1. Jean Alias- La via férrea. Madrid 1994. Ed. EYRROLL. Pp. 147.

2. Hugo Togno. Ferrocarriles. Мексика. 1970. Рp. 59.

3. Fernando Rey Valderrama. Ведение комплексной системы массового транспорта. Районное Начальство // Ciencia e ingeniería neogranadina No 12. 2002. Pp. 70.

4. Fernando Rey Valderrama. Ведение комплексной системы массового транспорта. Районное Начальство // Ciencia e ingeniería neogranadina No 12. 2002. Pp. 71.

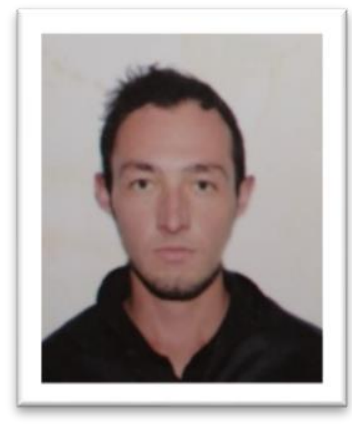

About the author: Juan Pablo Escobar, Civil Engineer with handling of structural and architectural design software, design assisted by BIM technology, experience as an engineering assistant, knowledge in railway and airport infrastructure acquired in the UNLP of Argentina. I am currently working on a master's degree in design and construction of large structures in the Russian language in the city of Moscow. Person with high sense of responsibility, punctual, dedicated, applied, practical, excellent interpersonal relations, entrepreneur in the work in group and decision making. 